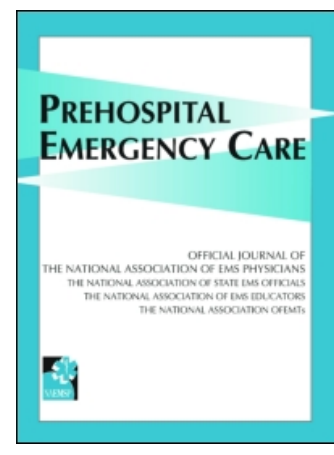

Prehospital Emergency Care

ISSN: 1090-3127 (Print) 1545-0066 (Online) Journal homepage: http://www.tandfonline.com/loi/ipec20

\title{
National Prehospital Evidence-Based Guidelines Strategy: A Summary for EMS Stakeholders
}

\section{Christian Martin-Gill MD, MPH, Joshua B. Gaither MD, Blair L. Bigham MD,} MSc, ACPf, J. Brent Myers MD, MPH, Douglas F. Kupas MD \& Daniel W. Spaite $M D$

To cite this article: Christian Martin-Gill MD, MPH, Joshua B. Gaither MD, Blair L. Bigham MD, MSc, ACPf, J. Brent Myers MD, MPH, Douglas F. Kupas MD \& Daniel W. Spaite MD (2016): National Prehospital Evidence-Based Guidelines Strategy: A Summary for EMS Stakeholders, Prehospital Emergency Care, DOI: 10.3109/10903127.2015.1102995

To link to this article: http://dx.doi.org/10.3109/10903127.2015.1102995

+ View supplementary material $匚$

曲 Published online: 25 Jan 2016.

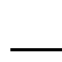

Submit your article to this journal $๘$

Џ Article views: 55

Q View related articles ¿

View Crossmark data \lceil 


\title{
National Prehospital Evidence-Based Guidelines Strategy: A SUMMARY FOR EMS STAKEHOLDERS
}

\author{
Christian Martin-Gill, MD, MPH, Joshua B. Gaither, MD, Blair L. Bigham, MD, MSc, ACPf, \\ J. Brent Myers, MD, MPH, Douglas F. Kupas, MD, Daniel W. Spaite, MD
}

ABSTRACT

Multiple national organizations have recommended and supported a national investment to increase the scientific evidence available to guide patient care delivered by Emergency Medical Services (EMS) and incorporate that evidence directly into EMS systems. Ongoing efforts seek to develop, implement, and evaluate prehospital evidence-based guidelines (EBGs) using the National Model Process created by a multidisciplinary panel of experts convened by the Federal Interagency Committee on EMS (FICEMS) and the National EMS Advisory Council (NEMSAC). Yet, these and other EBG

Received June 12, 2015 from University of Pittsburgh Department of Emergency Medicine, Pittsburgh, Pennsylvania (CMG); University of Arizona, Tucson, Arizona (JBG); St. Michael's Hospital and University of Toronto, Toronto, Ontario, Canada (BLB); University of North Carolina, Emergency Medicine, Chapel Hill, North Carolina (JBM); Geisinger Health System, Emergency Medicine, Danville, Pennsylvania (DFK); University of Arizona, Emergency Medicine, Tucson, Arizona (DWS). Revision received September 22, 2015; accepted for publication September 23, 2015.

Blair Bigham is a consultant for MedicAlert Foundation of Canada. Joshua B. Gaither received a grant from NIH/NINDS (Traumatic Brain Injury) and Philips Corporation \& Zoll Medical (Real-Time Audio-Visual Feedback in CPR). Daniel W. Spaite received a grant from NIH/NINDS (EPIC Study, Implementing the EMS EBGs statewide in Arizona and other research support from Medtronic Foundation, Implementing OHCA EBGs statewide in Arizona).

The National Prehospital EBG Strategy was developed by the National Association of EMS Physicians as part of a cooperative agreement with the National Highway Traffic Safety Administration (NHTSA) of the U.S. Department of Transportation (DOT) (DTNH22-13-H-00435). Funding was provided by NHTSA and the Emergency Medical Services for Children (EMSC) program of the Health Resources and Services Administration. The authors would like to thank Catherine S. Gotschall, $\mathrm{ScD}$, for her detailed input on the Strategy as the Contracting Office's Representative.

The opinions, findings and conclusions expressed in this publication are those of the authors and not necessarily those of NHTSA or the DOT. The United States Government assumes no liability for its content or use thereof. If trade or manufacturer's names or products are mentioned, it is because they are considered essential to the object of the publication and should not be construed as an endorsement. The United States Government does not endorse products or manufacturers.

Address correspondence to Christian Martin-Gill, University of Pittsburgh, Department of Emergency Medicine, Iroquois Bldg, Suite 400A, 3600 Forbes Ave, Pittsburgh, PA 15261, USA. E-mail: martingillc2@upmc.edu

doi: 10.3109/10903127.2015.1102995 efforts have occurred in relative isolation, with limited direct collaboration between national projects, and have experienced challenges in implementation of individual guidelines. There is a need to develop sustainable relationships among stakeholders that facilitate a common vision that facilitates EBG efforts. Herein, we summarize a National Strategy on EBGs developed by the National Association of EMS Physicians (NAEMSP) with involvement of 57 stakeholder organizations, and with the financial support of the National Highway Traffic Safety Administration (NHTSA) and the EMS for Children program. The Strategy proposes seven action items that support collaborative efforts in advancing prehospital EBGs. The first proposed action is creation of a Prehospital Guidelines Consortium (PGC) representing national medical and EMS organizations that have an interest in prehospital EBGs and their benefits to patient outcomes. Other action items include promoting research that supports creation and evaluates the impact of EBGs, promoting the development of new EBGs through improved stakeholder collaboration, and improving education on evidence-based medicine for all prehospital providers. The Strategy intends to facilitate implementation of EBGs by improving guideline dissemination and incorporation into protocols, and seeks to establish standardized evaluation methods for prehospital EBGs. Finally, the Strategy proposes that key stakeholder organizations financially support the Prehospital Guidelines Consortium as a means of implementing the Strategy, while together promoting additional funding for continued EBG efforts. Key words: clinical practice guidelines; evidencebased medicine; emergency medical services

PREHOSPITAL EMERGENCY CARE 2016; Early Online:1-9

\section{INTRODUCTION}

The creation of evidence-based guidelines (EBGs) for prehospital care has been described as "a process whose time has come." ${ }^{11}$ The 2001 publication of the National Emergency Medical Services (EMS) Research Agenda identified a need for a national investment in EMS research infrastructure and the application of scientific evidence to improve patient care. ${ }^{2}$ In the landmark 2006 report "Emergency Medicine at the Crossroads," the Institute of Medicine's Committee on the Future of Emergency Care in the United States Health System recommended the development of evidence-based protocols for the treatment of patients cared for by emergency medical services (EMS). ${ }^{3}$ Multiple other countries have also developed EMS research agendas. ${ }^{4}$ Yet, over the past decade, the creation of robust EBGs has only occurred for a limited set of medical conditions. 
EMS medicine is a complex clinical practice involving a variety of environments and a wide range of medical conditions. While the field suffers from a paucity of evidence, substantial advances in EMS research continuously occur and have the potential to markedly improve outcomes. ${ }^{4-7}$ Knowledge translation is a challenging task for individual prehospital practitioners and medical directors. Clinical guidelines serve to synthesize available evidence and bridge the gap between science and clinical practice. Although there is limited research on the direct benefits of existing prehospital EBGs, rigorously developed clinical guidelines are widely utilized in other medical fields and have been linked to improvements in patient care. ${ }^{8-13}$ EBGs do not equate to rigid protocols that lack flexibility for individual EMS systems. Instead, EBGs provide a framework that can improve patient care by reducing variability in care delivery; improving the quality of clinical decisions; and contributing to efficiency, cost containment, and improved patient outcomes. ${ }^{14}$ In combination with evidencebased performance measures for EMS, ${ }^{15}$ the use of EBGs provide a unique opportunity to improve care for time-dependent conditions such as trauma, stroke, myocardial infarction, and cardiac arrest, fostering innovation in managing these and other high-risk conditions.

Recognizing the potential opportunity to enhance prehospital care, improve health outcomes and optimize resource utilization by EMS systems for persons injured in motor vehicle crashes or who have other health emergencies, NHTSA, in partnership with the Health Resources and Services Administration's (HRSA) EMS for Children (EMSC) Program, provided funding for several efforts to develop and implement EBGs. ${ }^{1,16}$ NHTSA, in collaboration with the Federal Interagency Committee on EMS (FICEMS) and the National EMS Advisory Council (NEMSAC), convened a diverse group of EMS stakeholders and experts who developed a National Model Process for developing, implementing, and evaluating EBGs. ${ }^{17}$ This Model Process has since been used to develop guidelines for pediatric seizures, pain management, the use of air ambulances to transport trauma patients, and external hemorrhage control. ${ }^{16,18-22}$ In addition, there are multiple ongoing efforts led by national organizations and research institutions, largely funded by Federal agencies, working on a variety of activities that support prehospital EBGs.

Recently, NEMSAC and FICEMS provided recommendations to support development and use of EBGs. ${ }^{23,24}$ These recommendations emphasized the importance of forming relationships among EMS stakeholders and the need for EMS research and education that will hasten the process of EBG development and implementation. NHTSA subsequently awarded a cooperative agreement to NAEMSP, with supple- mental funding from EMSC, to develop a National Strategy on Prehospital EBGs. This Strategy aimed to engage EMS stakeholders at all levels to identify sustainable approaches to promote the development, implementation, and evaluation of EBGs. Herein, we summarize the Strategy created by a steering committee from NAEMSP in collaboration with representatives of 57 other organizations representing all aspects of EMS and prehospital care (the full Strategy is available as Data Supplement 1).

\section{MethodS}

In August 2013, NAEMSP convened a steering committee comprising EMS physicians, researchers, and field providers from the United States and Canada. A representative from NHTSA provided technical support to the steering committee. The steering committee identified stakeholders, facilitated discussion among participants during an in-person meeting and conference calls, provided input on incorporating stakeholder suggestions into the Strategy, and developed a series of action items that addressed the following Strategy objectives:

1. Identify national, state, tribal, and local stakeholder organizations whose missions include improving prehospital clinical care;

2. Develop mechanisms to build and sustain relationships among these identified organizations, encouraging their participation in EBG efforts;

3. Expand existing opportunities to conduct scientific research supporting EBGs and opportunities to present research findings to diverse audiences;

4. Identify ways to promote interest in EBG development among researchers, emergency physicians, prehospital care providers, and EMS officials;

5. Identify pathways to promote the incorporation of new EBGs into EMS education standards, the National Scope of Practice Model, and continued competency training; as well as stimulate related training resources for use by EMS educators and by state, local, and tribal EMS agencies;

6. Promote the dissemination and implementation of EBGs;

7. Identify ways to promote standardized evaluation of EBG effectiveness, including assessing their implementation and their impact on patientcentered outcomes and EMS resource utilization; and

8. Promote funding for future EBG activities.

Identifying stakeholders followed a stepwise process that engaged representatives from national medical organizations, EMS organizations, research institutions, 


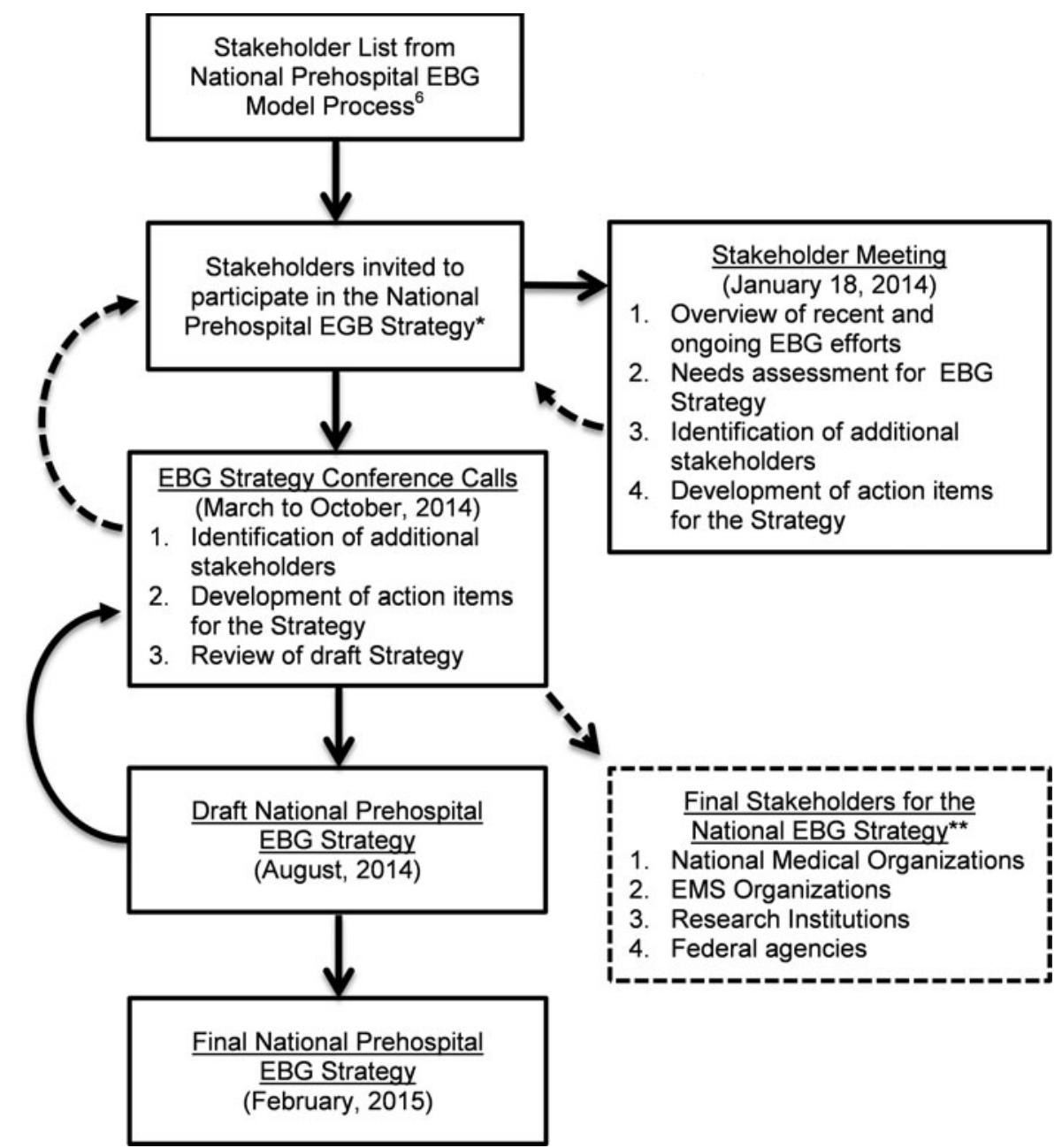

FIGURE 1. Stakeholder recruitment and participation in the EBG strategy. * Recruitment of stakeholders represented by dashed lines. ** List of individual stakeholders available as Data Supplement 2.

and Federal agencies (Figure 1). Snowball sampling 25 was used as part of an iterative process whereby the leaders and representatives of each organization were asked to recommend additional stakeholders that should be included. The final list of stakeholders that participated in the Strategy is provided in Data Supplement 2. This group developed seven action items and corresponding objectives that comprise the Strategy and are summarized as shown in the following section.

\section{Discussion}

\section{Action Item \#1: Create a Prehospital Guidelines Consortium}

The Strategy proposes the creation of a Prehospital Guidelines Consortium (PGC) as a mechanism to facilitate improved communication among organizations working on EMS EBG-related projects, connect stakeholders with researchers, and improve the cohesiveness and efficiency of EBG efforts (Figure 2). This Consortium will include national EMS and medical organizations and be led by an Executive Committee of representatives from key organizations that are most actively involved in prehospital EBG efforts (Figure 3). Organizations forming the Executive Committee will provide funding for the establishment and ongoing meetings of the Consortium. Activities of the PGC will include:

1. Facilitating improved communication related to EBG projects, research, and education among all stakeholders;

2. Creating and maintaining documents detailing ongoing gaps in EBG knowledge to inform future EBG-related research and EBG development projects;

3. Reviewing EBG-related project plans and draft documents, with the authority to officially endorse projects; 


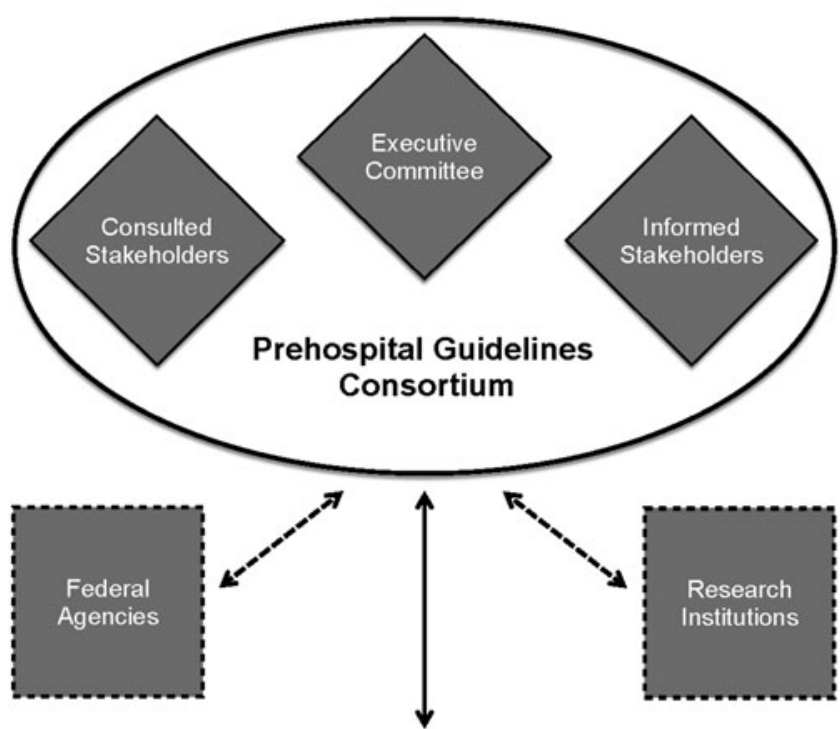

Consortium Objectives

1. Promote EMS EBG development research

2. Promote development of EMS EBGs

3. Promote education related to EMS EBGs

4. Promote implementation of EMS EBGs

5. Promote standardized evaluation methods for EMS EBGs

6. Promote funding for the development of EMS EBGs

Recommended Executive Committee Members: AMPA, AAEM, AAP, ACEP, ACOEP ACS-COT, AHA, NAEMSE, NAEMSP, NAEMT, NASEMSO, NREMT, SAEM

Figure 2. The Prehospital Guidelines Consortium. Air Medical Physicians Association (AMPA), American Academy of Emergency Medicine (AAEM), American Academy of Pediatrics (AAP), American College of Emergency Physicians (ACEP), American College of Osteopathic Emergency Physicians (ACOEP), American College of Surgeons Committee on Trauma (ACS-COT), American heart Association (AHA), National Association of EMS Educators (NAEMSE), National Association of EMS Physicians (NAEMSP), National Association of EMTs (NAEMT), National Association of EMS officials (NASEMSO), National Registry of EMTs (NREMT), Society for Academic Emergency Medicine (SAEM). Liaisons to the Prehospital Guideline Consortium represented by dashed lines.

- Voting members will include an Executive Committee of representatives from organizations that are most closely and actively involved in prehospital EBG efforts. Members of the Executive Committee will:

- Lead workgroups of the PGC that facilitate carrying out other action items of the Strategy;

- Elect a chair to serve a 2-year term and other officers as needed; and

- Provide funding for the PGC.

- Non-voting members of the PGC will include representatives of other EMS stakeholder organizations. These members include consulted and informed organizations as outlined in the Strategy and will:

- Participate in meetings of the PGC and

- Participate in workgroups that facilitate carrying out other action items of the Strategy.

- Research institutions, Federal agencies, and other relevant organizations may designate liaisons to interact with the PGC and participate in its meetings.

- Recommended initial workgroups include:
- Research
○ Development
- Education
- Implementation and Evaluation
- Funding

Figure 3. Proposed structure of the Prehospital Guidelines Consortium. 
4. Disseminating newly-created EBGs to stakeholders;

5. Maintaining a repository of existing prehospital EBGs and facilitating access by end-users;

6. Liaising with other organizations involved in EBG activities; and

7. Coordinating the implementation of the Strategy.

Creating new EBGs is a labor-intensive task, requiring ongoing funding and expertise specific to each EBG topic. The Consortium will provide overall coordination but will not create individual guidelines, perform or direct the performance of primary research in EMS medicine, or perform evaluations of individual guidelines. Independent groups, through separate funding mechanisms, will continue to lead this work, yet will be supported by the structure of the PGC and the collaboration that this new organization and its representatives can provide.

\section{Action Item \#2: Promote Research Supporting Prehospital Evidence-Based Guidelines}

The availability of scientific evidence on which to base recommendations is essential to the development of EBGs, yet limited research exists to guide EMS care. Prior efforts have identified specific areas of EMS that need further research, and include the National EMS Research Agenda ${ }^{2}$ and the Gap Analysis of EMSRelated Research, ${ }^{26}$ among others. ${ }^{27-30}$ Additionally, the Proceedings of the Implementation Symposium of the National EMS Research Agenda ${ }^{31}$ provided specific methods to move the recommendations forward. These included improving training opportunities for EMS researchers, increasing funding sources for EMS research, and facilitating the integration of research into practice.

Research that evaluates the effectiveness of EBGs is sorely needed and should focus on:

1. Assessing the impact of specific guidelines on patient-centered outcomes such as: survival, improving physiology, limiting disability, alleviating discomfort, patient satisfaction, and costeffectiveness, ${ }^{28}$ as well as EMS system resource utilization.

2. Evaluating the feasibility and efficiency of implementing EBGs into practice, focusing on implementation science (the interface between science and the clinical environment where it can be applied), knowledge translation (achieving awareness and agreement on use of EBGs), and behavioral change theory (evaluation of the science on behavior and change management as it relates to providers' implementation of EBGs). ${ }^{32-35}$
3. Assessing the use of best practices (as determined through consensus of an expert panel), such as implementation "toolkits" and other standardized implementation techniques.

\section{Action Item \#3: Promote Development of Prehospital Evidence-Based Guidelines}

The creation of EBGs, such as those recently developed following the Model Process, ${ }^{18-22}$ is a resource and time-intensive endeavor. These individual efforts require assembling a panel of experts and stakeholders, providing training to the panel to consistently apply evidence evaluation methodologies (e.g., the Grading of Recommendations, Assessment, Development, and Evaluation [GRADE] tool), ${ }^{36,37}$ developing the research questions, performing comprehensive literature reviews, and developing recommendations for the guidelines. Identifying ways to streamline this process, fostering collaboration among groups, and supporting EBG efforts through resource sharing has the potential to increase the pace at which EBGs are developed and revised. For example, creating mutually-available comprehensive literature reviews and other intermediary work products increases the likelihood that each product will be of high quality and consistent with related guidelines even when developed by independent groups.

Future steps in promoting EBG development should include:

1. Performing a gap analysis that identifies areas of EMS that would most benefit from EBGs, beginning with a review of existing EBGs and the National Association of State EMS Officials (NASEMSO) Model Clinical Guidelines document. ${ }^{38}$ Rigorous tools such as the GRADE $\operatorname{grid}^{39}$ and the guidelines checklist of Schunemann et al. ${ }^{40}$ should be utilized to determine when it is appropriate to proceed with guideline development on a particular topic. Resources that may inform the need for guideline development include the National EMS Information System (NEMSIS) ${ }^{41}$ and the Practice Analysis being performed by the National Registry of Emergency Medical Technicians. Attention to vulnerable populations should also be considered, such as pediatric patients and other patient groups where variability in care may be greatest or where the knowledge-practice gap is large.

2. Ensuring that guidelines are developed using processes consistent with the Institute of Medicine Standards for Developing Trustworthy Clinical Practice Guidelines, ${ }^{42}$ the National Prehospital EBG Model Process, ${ }^{1,17}$ and rigorous 
methodologies such as GRADE. ${ }^{36,37,39}$ These processes ensure that whether the level of scientific evidence is weak or strong, guidelines appropriately represent the available evidence and correspondingly grade recommendations.

3. Institutions with methodological expertise, such as the Evidence-based Practice Centers supported by the Agency for Healthcare Research and Quality, should be considered for performing systematic literature reviews, thus avoiding the timeconsuming process of teaching a content expert panel about scientific literature review for each individual EBG effort. ${ }^{22}$ This approach was used successfully in the development of the recently published external hemorrhage control EBG, ${ }^{11}$ which was developed by the American College of Surgeons using a systematic literature review funded by NHTSA.

4. The typical "life-cycle" of an EBG should be established whereby each EBG is comprehensively reviewed and revised at a standardized frequency. This should optimally occur through a living document that is regularly updated as new data become available, with revisions occurring at least every 5 years, consistent with requirements of the National Guideline Clearinghouse. ${ }^{43}$

\section{Action Item \#4: Improve Education Related to Prehospital Evidence-Based Guidelines}

Principles of evidence-based medicine have historically been lacking in EMS provider education. Students may not be exposed to the evidence supporting current EMS practices or the concept that EMS practice continuously evolves through research and translation of evidence. An essential step to foster the use of EBGs is enhancing EMS provider knowledge of evidencebased medicine at all levels of EMS provider certification and recertification. This must be accompanied by a paradigm shift to increase exposure to research principles in initial and continuing EMS education. ${ }^{1,23}$ This exposure should be progressive, with introduction at the lowest provider levels and a more intensive evidence-based medicine curriculum at the paramedic level.

Specific actions for improving education related to prehospital EBGs should include:

1. Incorporating basic principles of research, EBG methodology, and examples of current EBGs into initial and continuing education curricula, including: the National EMS Core Content, ${ }^{44}$ National EMS Education Standards, ${ }^{45}$ and textbook or online EMS educational content.

2. Incorporating EBG concepts into national and state-based EMS certification and recertification examinations, with the support of accrediting bodies for EMS education.

3. Making available educational opportunities highlighting the development and importance of EBGs to EMS educators, managers, regulators, physicians, and affiliated staff who are in a position to impact the implementation and use of EBGs.

4. Developing and enhancing pathways for prehospital providers to pursue academic careers.

\section{Action Item \#5: Facilitate the Implementation of Prehospital Evidence Based Guidelines}

Implementation of new evidence-based practices is a critical challenge for EMS systems ${ }^{19,35,46}$ and other healthcare settings. ${ }^{32,47-49}$ There is a general lack of knowledge regarding which tools are best used for the successful implementation of guidelines in EMS systems at the local, regional, state, tribal, and national levels. Concerns over clinical guidelines leading to "cook-book medicine" can also contribute to delays in adoption. ${ }^{14}$ However, EBGs based on GRADE or similar methodology for evidence appraisal and grading of recommendations provide a structured framework from which EMS medical directors and practitioners can develop adaptive protocols that take into account differences in practice environments, available medications, equipment, geography, training capabilities, provider levels, and scope of practice. Other critical components for successful implementation of an EBG include: engaging stakeholders, use of structured evaluation tools to identify barriers to implementation, the creation of sample tools to assist local EMS agencies in the implementation process, and transparency in the guideline development process. ${ }^{17}$

Recommended objectives to facilitate implementation of prehospital EBGs include:

1. Establishing a structured mechanism for stakeholders who will be in a position to implement guidelines to systematically review, provide input, and vet guidelines throughout the process of development.

2. Developing a systematic process for EBG dissemination that includes initial distribution: a) through NASEMSO to state EMS offices and then to regional and local EMS systems, b) through NAEMSP to EMS medical directors, c) through the Committee on Accreditation of Educational Programs for the Emergency Medical Services Professionals (CoAEMSP) and the National Association of EMS Educators (NAEMSE) to training centers for incorporation in EMS educational curricula, and 4) through other PGC members. 
3. Promoting the use of structured evaluation tools that identify challenges to and feasibility of implementation [e.g., Appraisal of Guidelines, Research and Evaluation (AGREE) and the Guideline Implementability Appraisal (GLIA) tool]..$^{50,51}$

4. Developing clinical tools such as sample protocols, educational content including a guidelines synopsis, ${ }^{52}$ and training materials that can help in local implementation.

5. Ensuring there is clarity on any potential conflicts of interest as guidelines are developed to addressing perceptions of bias that can hamper implementation of evidence-based recommendations.

6. Establishing a concrete timetable for expected implementation and revision of guidelines, in order to facilitate implementation planning and reeducation.

7. Promoting research on specific implementation tools, including the evaluation of implementation toolkits, ${ }^{53}$ technological adjuncts such as feedback devices, ${ }^{54}$ and cognitive and behavioral models that explain and enhance compliance with guidelines. ${ }^{55}$

\section{Action Item \#6: Establish Standardized Evaluation Methods for Prehospital Evidence-Based Guidelines}

Evaluating the effectiveness and outcomes of EBGs is an essential component of the National Prehospital Evidence-Based Guideline Model Process ${ }^{17}$ and should be an integral part of all guideline projects. Future efforts for EBG evaluation should include:

1. Determining whether individual guideline implementation was successful, if it improved outcomes, and whether the EBG is being continuously updated as new evidence is generated.

2. Use of uniform data definitions and repositories, such as the NEMSIS, in order to assist in standardizing comparisons among EMS systems and facilitate a wider evaluation of the EBG. ${ }^{41,56,57}$

3. Disseminating findings of EBG assessments to funding agencies and guideline developers to assist with the refinement of future guidelines.

\section{Action Item \#7: Promote Funding for the Development, Implementation, and Evaluation of Prehospital Evidence Based Guidelines}

Continued funding for EBG development is essential and will dictate the speed at which a comprehensive set of guidelines is created. In its 2012 Summary Rec- ommendations, the National EMS Advisory Council recommended the creation of Federal funding sources specifically for EMS research to support the development of EBGs, as well as the creation of center(s) of excellence for EMS EBG development. ${ }^{23}$ An Evidencebased Practice Center ${ }^{58}$ focused on EMS medicine ${ }^{59}$ would certainly be the optimal mechanism to advance the development of these EBGs, but this would require substantial funding. In the short term, funding of EBG efforts can be supported by:

1. Supporting the creation of the Prehospital Guidelines Consortium through funding from key stakeholders that will form the Executive Committee of the Consortium, thus beginning the implementation of the EBG Strategy in a sustainable manner.

2. Promoting continued and additional support from Federal partners and other EMS and medical organizations to fund new projects to develop, implement, and evaluate specific, high priority guidelines.

\section{Evaluation Plan for the Prehospital Evidence-based Guidelines Strategy}

The evaluation of this strategy at regular intervals (e.g., every 5 years $)^{43}$ is just as important as the evaluation of EBGs to ensure their effectiveness. A proposed plan for evaluation of the Strategy includes measures of context (aspects of the social, political, and economic environment that may influence implementation of the Strategy); recruitment (the methods by which organizations are included in the implementation of the Strategy); reach (identifying the target audience of EMS stakeholders and the degree to which the Strategy is reaching those it was intended to affect); dose delivered (the amount of the Strategy interventions that were implemented); dose received (the extent to which the target audience [who is being asked to develop, utilize, or evaluate EBGs] actively engage with, interact with, are receptive to, and/or use materials or recommended resources); and fidelity (the extent to which the Strategy is being carried out as planned). ${ }^{60}$

\section{CONCLUSION}

Recent recommendations from NEMSAC and the objectives of the FICEMS Strategic Plan support the creation of a sustainable National Strategy for the development, implementation, and evaluation of prehospital evidence-based guidelines. Creation of a Prehospital Guidelines Consortium would improve communication among stakeholders and EBG project groups, improve efficiency of EBG development, and support more widespread adoption of EBGs. Other 
elements of this Strategy promote EMS research that supports EBG development and education of EMS providers in evidence-based medicine, two of several components that may lead to improved implementation of EBGs. By advancing this Strategy, we seize an opportunity to elucidate and implement best practices for patients and to transform prehospital care.

\section{References}

1. Wright JL. Evidence-based Guidelines for prehospital practice: a process whose time has come. Prehosp Emerg Care. 2014;18(S1):1-2.

2. Sayre MR, White LJ, Brown LH, McHenry SD, National EMSAWT. National EMS research agenda. Prehosp Emerg Care. 2002;6:S1-43.

3. Institute of Medicine (U.S.). Committee on the Future of Emergency Care in the United States Health System. Emergency Medical Services at the Crossroads. Washington, DC: National Academies Press, 2007.

4. Jensen JL, Bigham BL, Blanchard IE, et al. The Canadian National EMS Research Agenda: a mixed methods consensus study. CJEM. 2013;15:73-82.

5. National Highway Traffic Safety Administration. National EMS Research Agenda. 2001. Available at: www.ems.gov/research.html. Accessed July 22, 2015.

6. MacFarlane $\mathrm{C}$. The advances and evidence base for prehospital care. Emerg Med J. 2003;20:114-15.

7. Smith E, Jennings $\mathrm{P}, \mathrm{McD}$ onald $\mathrm{S}$, MacPherson $\mathrm{C}, \mathrm{O}^{\prime}$ Brien $\mathrm{T}$, Archer F. The Cochrane Library as a resource for evidence on out-of-hospital health care interventions. Ann Emerg Med. 2007;49:344-50.

8. Cheng $\mathrm{SH}$, Wang $\mathrm{CJ}$, Lin JL, et al. Adherence to quality indicators and survival in patients with breast cancer. Med Care. 2009;47:217-25.

9. Boland GM, Chang GJ, Haynes AB, et al. Association between adherence to National Comprehensive Cancer Network treatment guidelines and improved survival in patients with colon cancer. Cancer. 2013;119:1593-601.

10. Fritz JM, Cleland JA, Brennan GP. Does adherence to the guideline recommendation for active treatments improve the quality of care for patients with acute low back pain delivered by physical therapists? Med Care. 2007;45:973-80.

11. Udovcic M, Castro JC, Apsey HA, Stearns JD, Schlinkert RT, Cook CB. Guidelines to improve perioperative management of diabetes mellitus: assessment of the impact of change across time. Endocr Pract. 2015;21:1026-34.

12. Lugtenberg M, Burgers JS, Westert GP. Effects of evidencebased clinical practice guidelines on quality of care: a systematic review. Qual Saf Health Care. 2009;18:385-92.

13. Roberts ET, DuGoff EH, Heins SE, et al. Evaluating clinical practice guidelines based on their association with return to work in administrative claims data. Health Serv Res. 2015. (Epub ahead of print Sept 14, 2015).

14. Andrews EJ, Redmond HP. A review of clinical guidelines. Br J Surg. 2004;91:956-64.

15. Myers JB, Slovis CM, Eckstein M, et al. Evidence-based performance measures for emergency medical services systems: a model for expanded EMS benchmarking. Prehosp Emerg Care. 2008;12:141-51.

16. Progress on evidence-based guidelines for prehospital emergency care. Office of Emergency Medical Services, National Highway Traffic Safety Administration, 2013. Available at: www.nhtsa.gov/staticfiles/nti/pdf/811643.pdf. Accessed June 7, 2015.
17. Lang ES, Spaite DW, Oliver ZJ, et al. A national model for developing, implementing, and evaluating evidence-based guidelines for prehospital care. Acad Emerg Med. 2012;19:201-09.

18. Shah MI, Macias CG, Dayan PS, et al. An evidence-based guideline for pediatric prehospital seizure management using GRADE methodology. Prehosp Emerg Care. 2014;18(S1):15-24.

19. Brown KM, Hirshon JM, Alcorta R, et al. The implementation and evaluation of an evidence-based statewide prehospital pain management protocol developed using the national prehospital evidence-based guideline model process for emergency medical services. Prehosp Emerg Care. 2014;18(S1):45-51.

20. Brown KM, Macias CG, Dayan PS, et al. The development of evidence-based prehospital guidelines using a GRADE-based methodology. Prehosp Emerg Care. 2014;18(S1):3-14.

21. Thomas SH, Brown KM, Oliver ZJ, et al. An evidence-based guideline for the air medical transportation of prehospital trauma patients. Prehosp Emerg Care. 2014;18(S)1:35-44.

22. Bulger EM, Snyder D, Schoelles K, et al. An evidence-based prehospital guideline for external hemorrhage control: American College of Surgeons Committee on Trauma. Prehosp Emerg Care. 2014;18:163-73.

23. National Emergency Medical Services Advisory Council. National Emergency Medical Services Advisory Council Summary Report [2010-2012]. Report No. DOT HS 811 705. National Highway Traffic Safety Administration, Washington, DC, 2013. Available at: www.ems.gov/NEMSAC.htm. Accessed June 7, 2015.

24. Federal Interagency Committee on EMS. FICEMS Strategic Plan. Report No. DOT HA 811 990. National Highway Traffic Safety Administration, Washington, DC, 2014. Available at: http://www.ems.gov/ficems/plan.htm. Accessed June 7, 2015.

25. Goodman LA. Snowball sampling. Ann Math Stats 1961;32:148-70.

26. EMSC National Resource Center, Children's National Medical Center. Gap Analysis of EMS Related Research. Report to the Federal Interagency Committee on EMS. 2009. Available at: www.childrensnational.org/emsc. Accessed June 7, 2015.

27. Seidel JS, Henderson D, Tittle S, et al. Priorities for research in emergency medical services for children: results of a consensus conference. Ann Emerg Med. 1999;33:206-10.

28. Maio RF, Garrison HG, Spaite DW, et al. Emergency medical services outcomes project I (EMSOP I): prioritizing conditions for outcomes research. Ann Emerg Med. 1999;33:423-32.

29. Spaite DW, Maio R, Garrison HG, et al. Emergency Medical Services Outcomes Project (EMSOP) II: developing the foundation and conceptual models for out-of-hospital outcomes research. Ann Emerg Med. 2001;37:657-63.

30. Foltin GL, Dayan P, Tunik M, et al. Priorities for pediatric prehospital research. Pediatr Emerg Care. 2010;26:773-77.

31. Sayre MR, White LJ, Brown LH, McHenry SD, Implementation Symposium P. National EMS research agenda: proceedings of the implementation symposium. Acad Emerg Med. 2003;10:1100-08.

32. Cabana MD, Rand CS, Powe NR, et al. Why don't physicians follow clinical practice guidelines? a framework for improvement. JAMA. 1999;282:1458-65.

33. Grimshaw JM, Shirran L, Thomas R, et al. Changing provider behavior: an overview of systematic reviews of interventions. Med Care. 2001;39:II2-45.

34. Davis D, Evans M, Jadad A, et al. The case for knowledge translation: shortening the journey from evidence to effect. BMJ. 2003;327:33-35.

35. Bigham BL, Aufderheide TP, Davis DP, et al. Knowledge translation in emergency medical services: a qualitative survey of barriers to guideline implementation. Resuscitation. 2010;81:836-40. 
36. Guyatt GH, Oxman AD, Vist GE, et al. GRADE: an emerging consensus on rating quality of evidence and strength of recommendations. BMJ. 2008;336:924-26.

37. Schunemann HJ, Oxman AD, Brozek J, et al. GRADE: assessing the quality of evidence for diagnostic recommendations. Evid Based Med. 2008;13:162-63.

38. National Association of State EMS Officials. National Model EMS Clinical Guidelines. 2014. Available at: www.nasemso. org/Projects/ModelEMSClinicalGuidelines/index.asp. Accessed June 7, 2015.

39. Jaeschke R, Guyatt GH, Dellinger P, et al. Use of GRADE grid to reach decisions on clinical practice guidelines when consensus is elusive. BMJ. 2008;337:327-30.

40. Schunemann HJ, Wiercioch W, Etxeandia I, et al. Guidelines 2.0: systematic development of a comprehensive checklist for a successful guideline enterprise. CMAJ. 2014;186:E123-42.

41. Dawson DE. National Emergency Medical Services Information System (NEMSIS). Prehosp Emerg Care. 2006;10:314-16.

42. Institute of Medicine (U.S.). Committee on Standards for Developing Trustworthy Clinical Practice Guidelines., Graham R. Clinical practice guidelines we can trust. Washington, DC: National Academies Press, 2011.

43. National Guideline Clearinghouse. U.S. Department of Health and Human Services, Agency for Healthcare Research and Quality, 2015. Available at: www.guideline. gov/about/inclusion-criteria.aspx. Accessed June 7, 2015.

44. U.S. Department of Transportation, National Highway Traffic Safety Administration. National EMS Core Content, 2005. Available at www.ems.gov/EducationStandards.htm. Accessed June 7, 2015.

45. U.S. Department of Transportation, National Highway Traffic Safety Administration. National EMS Education Standards, 2009. Available at: www.ems.gov/EducationStandards.htm. Accessed June 7, 2015.

46. Bigham BL, Koprowicz K, Aufderheide TP, et al. Delayed prehospital implementation of the 2005 American Heart Association guidelines for cardiopulmonary resuscitation and emergency cardiac care. Prehosp Emerg Care. 2010;14:355-60.

47. Gagliardi AR, Brouwers MC, Palda VA, Lemieux-Charles L, Grimshaw JM. How can we improve guideline use? a conceptual framework of implementability. Implement Sci. 2011; 6:26.
48. Leape LL, Weissman JS, Schneider EC, Piana RN, Gatsonis C, Epstein AM. Adherence to practice guidelines: the role of specialty society guidelines. Am Heart J. 2003;145:19-26.

49. Casey DE, Jr. Why don't physicians (and patients) consistently follow clinical practice guidelines? JAMA. 2013;173:1581-83.

50. Brouwers MC, Kho ME, Browman GP, et al. AGREE II: advancing guideline development, reporting and evaluation in health care. CMAJ. 2010;182:E839-42.

51. Shiffman RN, Dixon J, Brandt C, et al. The GuideLine Implementability Appraisal (GLIA): development of an instrument to identify obstacles to guideline implementation. BMC Med Inform Decis Mak. 2005;5:23.

52. Cifu AS, Davis AM, Livingston EH. Introducing JAMA clinical guidelines synopsis. JAMA. 2014;312:1208-09.

53. National Association of State EMS Officials. Statewide implementation of an evidence-based guideline: General toolkit V2.1, 2014. Available at: www.nasemso.org/Projects/ ImplementationOfEBG/index.asp. Accessed June 7, 2015.

54. Hostler D, Everson-Stewart S, Rea TD, et al. Effect of real-time feedback during cardiopulmonary resuscitation outside hospital: prospective, cluster-randomised trial. BMJ. 2011;342:d512.

55. Pathman DE, Konrad TR, Freed GL, Freeman VA, Koch GG. The awareness-to-adherence model of the steps to clinical guideline compliance. the case of pediatric vaccine recommendations. Med Care. 1996;34:873-89.

56. Mears G. Emergency medical services information systems. N C Med J. 2007;68:266-67.

57. Mears G, Ornato JP, Dawson DE. Emergency medical services information systems and a future EMS national database. Prehosp Emerg Care. 2002;6:123-30.

58. Evidence-based Practice Centers (EPC) Program Overview. U.S. Department of Health and Human Services, Agency for Healthcare Research and Quality, 2015. Available at: www.ahrq. gov/research/findings/evidence-based-reports/overview. Accessed June 7, 2015.

59. Perina DG, Pons PT, Blackwell TH, et al. The core content of emergency medical services medicine. Prehosp Emerg Care. 2012;16:309-22.

60. Steckler AB, Linnan L. Process evaluation for public health interventions and research. 1st ed. San Francisco, CA: JosseyBass, 2002. 